\title{
Dynamic Data-Driven Fault Diagnosis of Wind Turbine Systems
}

\author{
Yu Ding ${ }^{1}$, Eunshin Byon ${ }^{1}$, Chiwoo Park ${ }^{1}$, Jiong Tang ${ }^{2}$, Yi Lu ${ }^{2}$, and Xin Wang ${ }^{2}$ \\ ${ }^{1}$ Texas A\&M University, College Station, TX 77843 \\ yuding@iemial. tamu .edu \\ ${ }^{2}$ University of Connecticut, Storrs, CT 06269 \\ jtang@engr.uconn.edu
}

\begin{abstract}
In this multi-university collaborative research, we will develop a framework for the dynamic data-driven fault diagnosis of wind turbines which aims at making the wind energy a competitive alternative in the energy market. This new methodology is fundamentally different from the current practice whose performance is limited due to the non-dynamic and non-robust nature in the modeling approaches and in the data collection and processing strategies. The new methodology consists of robust data pre-processing modules, interrelated, multi-level models that describe different details of the system behaviors, and a dynamic strategy that allows for measurements to be adaptively taken according to specific physical conditions and the associated risk level. This paper summarizes the latest progresses in the research.
\end{abstract}

\section{Introduction}

Wind turbines convert the kinetic energy of wind into the electrical energy, which provides a pollution-free source of electricity. Today wind power is considered the fastest growing energy source around the world. The key issue for all renewable energy utilizations is the cost and the marketability [1]. In the US, Class 6 sites (with average wind speeds of $6.7 \mathrm{~m} / \mathrm{s}$ at $10 \mathrm{~m}$ height) can in theory market electricity at prices of 3 to $4 \mathrm{c} / \mathrm{kWh}$, which, together with the tax credit $(1.7 \mathrm{c} / \mathrm{kWh})$, allows wind energy to compete with traditional energy sources. As more sites are developed, easily accessible new Class 6 sites are becoming less available. Emphasis has now been shifted to Class 4 wind sites $(5.8 \mathrm{~m} / \mathrm{s}$ at $10 \mathrm{~m}$ height), which cover vast areas of the Great Plains from northern Texas to the US-Canada border. Class 4 sites represent almost 20 times the developable wind resource of Class 6 sites. Currently the electricity at Class 4 sites can be generated at costs in the range of 5 to $6 \mathrm{c} / \mathrm{kWh}$. In order to position the wind energy as an attractive option, the Federal Wind Energy Program (FWEP) has set a goal to reduce the cost of electricity generated at Class 4 sites to $3 \mathrm{c} / \mathrm{kWh}$. One major hurdle to achieve this goal is the high cost for maintaining the wind turbines at remote areas, given the labors, time, and heavy-duty equipment involved. The FWEP's goal can be achieved only after the number of false alarms, the failure-caused down time, and the actual maintenance cost of wind turbines can be significantly reduced.

Clearly, a reliable, robust fault detection and diagnosis system plays a critical role in making the wind power more marketable. In a modern wind turbine, the most expensive and fault-prone components are the gearbox and the blades, especially for 
those low speed wind turbines operating at Class 4 sites. While researchers have pursued fault diagnosis for similar structures for decades, diagnosis of wind turbine components is unique and poses new challenge. First, these low speed wind turbines constantly operate under non-stationary conditions and involve very complicated gearboxes with multiple stages of gears to speed up a hundred times or more. Second, in order to increase the unit power conversion efficiency, the blades used are significantly longer, up to $70 \mathrm{~m}$ in length as compared to the $20 \mathrm{~m}$ ones currently installed in high speed wind turbines. For these blades with such enormous size and made of composite materials, traditional modal-information-based fault diagnosis is difficult to be implemented on-line and is insensitive to the dominant failure mode, namely the delamination within the layered composites [2].

The most challenging aspect of wind turbine fault diagnosis comes from the high requirements placed on the accuracy and credibility, owing to the cost-sensitive nature related to the FWEP's goal. The currently available signal processing and diagnosis methods are deterministic in nature, leading to a large number of false alarms [3]. In view of these challenges and the urgent need, in this project we develop a new framework for the effective and robust diagnosis of wind turbine systems using the dynamic data-driven methodology. This methodology, as illustrated in Fig. 1, consists of robust data pre-processing modules for highly sensitive feature extraction, interrelated models that describe different details of the system behaviors at multiple levels, and a dynamic strategy that allows the measurements to be adaptively taken according to the specific physical conditions and the associated risk level. In this paper, we summarize the progresses made in the research.

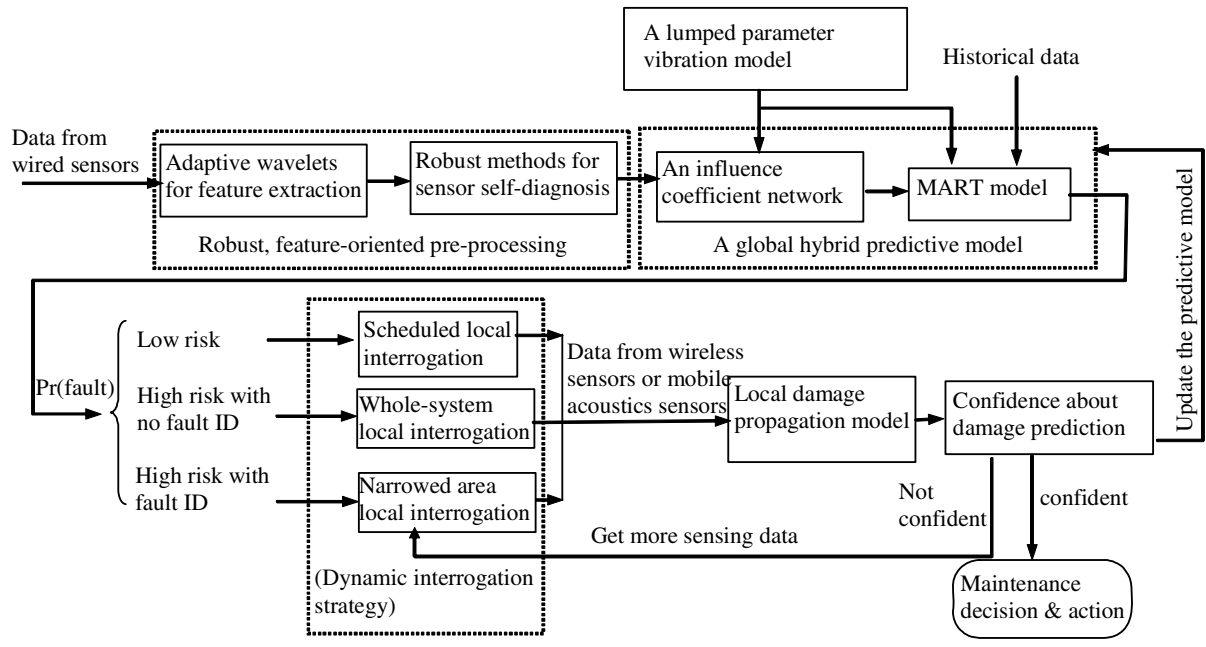

Fig. 1. Framework for dynamic, data-driven diagnosis of wind turbine systems

\section{Signal Pre-processing and Local-Level Detection}

At local level, we are concerned about the health conditions of the gearbox and the blades. For the monitoring of the gearbox, vibratory signals during the wind turbine 
operation collected by accelerometers will be used. For the monitoring of the blades, we mainly rely on an active damage interrogation scheme that uses embedded piezoelectric actuators to generate Lamb wave propagation in the blades. The wave propagation anomaly will indicate damage occurrence such as delamination.

The common feature of the aforementioned signals that are used for health monitoring is that they are non-stationary either due to the wind turbine operation, or due to the nature of the wave excitation. For such signals, it is better to choose timefrequency representations, among which wavelet analysis is particularly useful, to extract their critical features. Different from traditional Fourier transform-based frequency analysis, wavelet transforms lead to the flexibility in using narrow windows for the analysis of high-frequency content and wide windows for low frequencies. Newland [4] developed harmonic wavelet and its generalized form, whose wavelet levels represent non-overlapping frequency bands that can help interpret frequency contents and link detection results with physical meanings. Liu improved the generalized harmonic wavelet transform by applying an entropy-based algorithm for best basis selection, in the sense that it gives the sparsest representation of the signal [5]. Since the time-frequency interpretation is adaptive to specific signals, finding the common wavelet bases for a set of samples (signals) in the presence of noise requires extra improvement, which is the focus of our current study.

From the Fourier transform in the frequency domain

$$
W_{m n k}(\omega)=\left\{\begin{array}{cl}
\frac{1}{(n-m) 2 \pi} e^{-i \omega \frac{k}{n-m}} & m 2 \pi \leq \omega \leq n 2 \pi \\
0 & \text { otherwise }
\end{array}\right.
$$

Newland derived the family of generalized harmonic wavelets [4]

$$
w_{m n k}(t)=w_{m n}\left(t-\frac{k}{n-m}\right)=\frac{\exp \left[i n 2 \pi\left(t-\frac{k}{n-m}\right)\right]-\exp \left[i m 2 \pi\left(t-\frac{k}{n-m}\right)\right]}{(n-m) i 2 \pi t}
$$

where $m$ and $n$ are the level parameters, $0 \leq m \leq n$, and integer $k$ denotes the translation parameter within the level $(m, n)$. The advantage of harmonic wavelets is that signal analysis is restricted to specific frequency bands with known physical meanings, and these bands are represented by corresponding wavelet levels. A discrete algorithm is developed to calculate coefficients by computing the inverse discrete Fourier transform (IDFT) of successive blocks (each corresponds to a level $m, n$ ) of the Fourier coefficients of the input signal.

Liu treated each selection $\left\{\left(m_{0}, n_{0}\right),\left(m_{1}, n_{1}\right), \ldots,\left(m_{L-1}, n_{L-1}\right)\right\}$ as a partition of $\Omega=\left\{0,1, \ldots, N_{f}\right\}$ and developed a Shannon entropy-based algorithm to search a partition tree for the best partition (in the sense that the signal can be represented most sparsely) [5]. For any sequence $\mathbf{x}=\left\{x_{j}\right\}$, the Shannon entropy is defined as

$$
H(\mathbf{x})=-\sum_{j} p_{j} \log p_{j}
$$

where $p_{j}=\left|x_{j}\right|^{2} /\|\mathbf{x}\|^{2}$, and $p_{j} \log p_{j}$ is set as 0 if $p_{j}=0$. The entropy above is a measure of the sparsity and therefore we expect smaller entropy for a better partition of 
wavelet coefficients. We incorporate this technique to the pre-processing of Lamb wave-based damage detection in laboratory beam-type specimens. The iteration procedure for a sequence of 16 elements using a binary partition tree is illustrated in Fig. 2 (Phase II). For the initial partition, every single Fourier coefficient forms an 'initial subgroup', whose the Shannon entropy can be called initial entropy. Then in Step 1, every 2 successive Fourier coefficients form a 'Step 1 subgroup', whose entropy is calculated and compared with the corresponding sum of initial entropies. For the example in Fig. 2, the sum entropy of the 'initial subgroups' 1 and 2 is smaller than that of the 'Step 1 subgroup' 1. The former subgroups, instead of the latter, are therefore kept after the selection in Step 1. After the entire iteration, the features of the input signal will be highlighted by projecting samples onto those best basis functions.

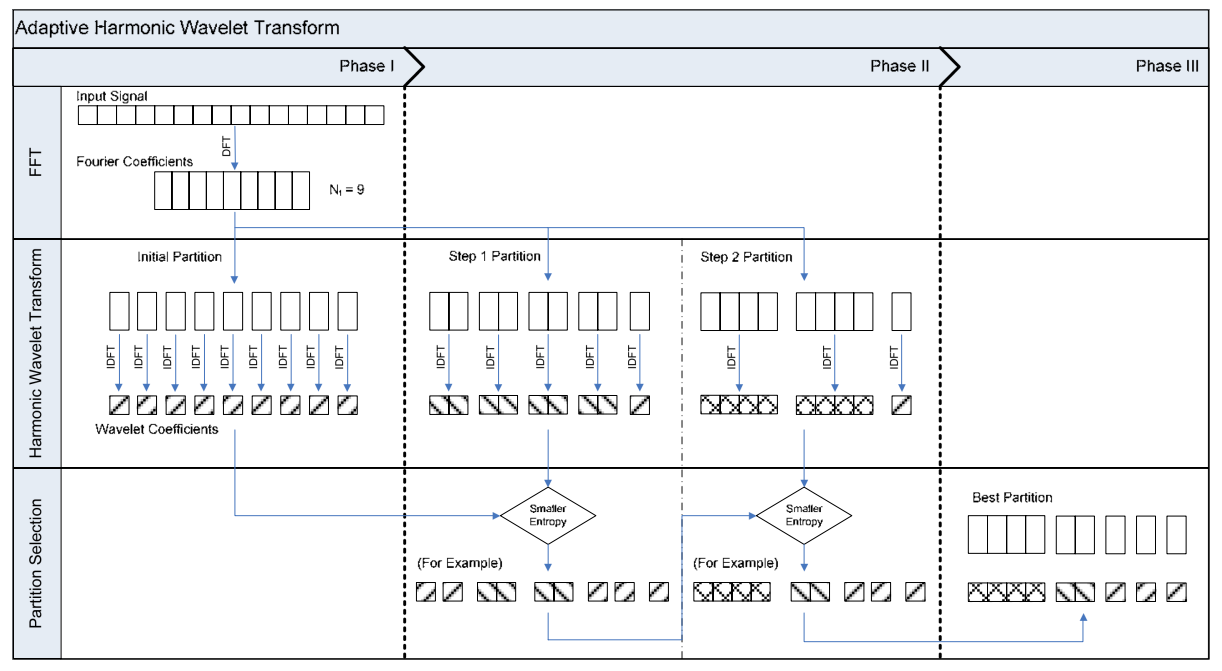

Fig. 2. Discrete-time adaptive harmonic wavelet transform using FFT/IFFT (N=16)

The above strategy of adaptive harmonic wavelet transform (AHWT) is signaldependent. Multiple signals from the same gearbox or blade may, however, lead to different wavelet basis partition simply due to the existence of noise. In order to build a baseline dataset, the detection algorithm requires a common wavelet basis set for all the samples. We hence extend the adaptive harmonic wavelet transform for multivariate applications. Each time series sample $\mathbf{s}_{l}=\left\{s_{l}(r), r=0,1, \ldots, N-1\right\}$ collected from the accelerometer or piezoelectric sensor is associated with a set of basis functions $\left\{\mathbf{w}_{\mathbf{m n k}}\right\}_{l}$, then all the $L$ samples are projected on $\left\{\mathbf{w}_{\mathbf{m n k}}\right\}_{l}$ to yield a matrix of wavelet coefficients $\left[\begin{array}{lll}\mathbf{a}_{\mathbf{1}} & \ldots & \mathbf{a}_{\mathbf{L}}\end{array}\right]_{l}$. The total Shannon entropy can be defined as $H\left(\left[\mathbf{a}_{1} \ldots \mathbf{a}_{\mathbf{L}}\right]_{l}\right)=H\left(\mathbf{a}_{\mathbf{1}}\right)+\ldots+H\left(\mathbf{a}_{\mathbf{L}}\right)$, where $l=1, \ldots, L$ (the number of samples). We select the common wavelet basis set $\left\{\mathbf{w}_{\mathbf{m n k}}\right\}_{u}$ such that the wavelet coefficients $\left[\mathbf{a}_{\mathbf{1}} \ldots\right.$ $\left.\mathbf{a}_{\mathbf{L}}\right]_{u}$ have the smallest total Shannon entropy, i.e.,

$$
u=\underset{l}{\arg \min } H\left(\left[\mathbf{a}_{\mathbf{1}} \ldots \mathbf{a}_{\mathbf{L}}\right]_{l}\right)
$$




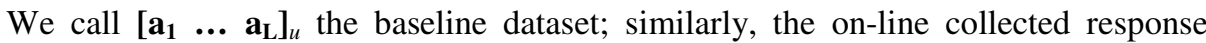
signal can be processed by applying the above procedure.

With the AHWT as basis for feature extraction, we may then use several statistical analysis tools for local-level detection. Principal component analysis (PCA) transforms a number of correlated variables into a smaller number of uncorrelated new variables called principal components. Consider a block of baseline data and let $\mathbf{C}$ be the corresponding covariance matrix. PCA yields an orthogonal (eigenvector) matrix $\mathbf{V}=\left[\begin{array}{lll}\mathbf{v}_{\mathbf{1}} & \ldots & \mathbf{v}_{\mathbf{K}}\end{array}\right]$ matrix and a diagonal (eigenvalue) matrix $\mathbf{D}=\operatorname{diag}\left(\lambda_{1}, \ldots, \lambda_{K}\right)$ such that $\mathbf{C V}=\mathbf{V D}$. The eigenvalues are arranged in descending order $\lambda_{j} \geq \lambda_{j+1}$. Introducing the effective rank $r k$ of $\mathbf{X}$ [6], we can discard the eigenvectors associated with $\lambda_{r k+1}, \ldots, \lambda_{K}$ and form a modified eigenvector matrix $\mathbf{V}_{\mathbf{m}}$. Here we choose $r k$ as the smallest number so that the accumulative energy is above a certain threshold ET\%,

$$
r k_{0}=\min r k \text { s.t. } \sum_{j=1}^{r k} \lambda_{j}>E T \% \sum_{j=1}^{K} \lambda_{j}
$$

given that the eigenvalues represent the distribution of the original energy among each of the eigenvectors. The local-level detection is facilitated by the Hotelling's $T^{2}$ analysis [7]. First, baseline data are sub-grouped and used to establish an upper control limit UCL ${ }^{1}$ under a certain confidence level $100(1-\alpha) \%$, where $\alpha$ indicates the error probability $(0<\alpha<1)$. Then in phase II, a distinction is made between the baseline and the online sensor data using a modified upper control limit $\mathrm{UCL}^{2}$. If any calculated $T^{2}$ value exceeds the phase II upper control limit, we may conclude, with
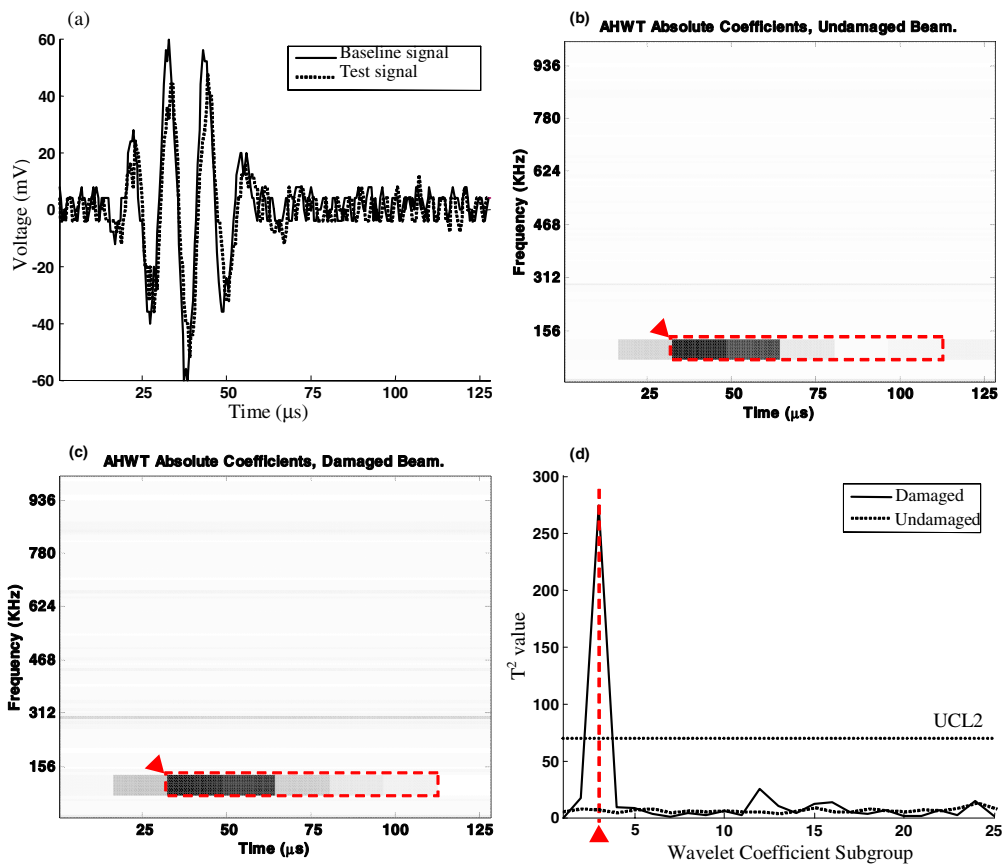

Fig. 3. Piezoelectric interrogation and wave signals: local-level data-driven signal processing 
confidence level $100(1-\alpha) \%$, that the structure is in its damaged state. Fig. 3 shows an example of successful detection of crack damage in a laboratory beam structure using piezoelectric-based damage interrogation.

\section{Dynamic Interrogation and Decision-Making}

We intend to develop a "risk-based dynamic decision making" policy, considering the loss that may be caused by undetected component failures and the cost of doing unnecessary maintenance work for normally operating components. In this dynamic procedure, we classify the sensor feedbacks and the subsequent actions into three levels: (1) the normal level, and thus operate as is, when no sign indicates any serious problems; (2) the alert level and then put the monitoring into the intensive care mode; (3) the alarm level, and thus dispatch the maintenance crew for on-site repairing. Under intensive care, one need update the sensor measurements more frequently, use advanced mobile sensors for obtaining additional information, and invoke computationally intensive modules for predicting failure modes with reduced uncertainty. So the intensive care mode will incur additional cost but will not be as expensive as dispatching the crew. The decision will be made dynamically by weighing a group of factors.

Factors need to be considered including the costs and risks associated with different actions to be taken (e.g., dispatch a maintenance crew or not), weather conditions now and in the near future which affects both the wind power generation, the severity of any existing mechanical problem, and the feasibility of a repairing mission, the loss caused by the disruption to power generation as the result of a major maintenance or as the result of an utter failure. A hidden Markov model [8] is being used to model the wind turbine's health status. Weather is an important condition for wind turbine operations because wind is the primary source for producing wind power [9]. Non-stationarity and irregularity in wind conditions induce the fatigues causing many component failures. The weather condition also affect the feasibility and cost of a repairing mission since doing a repair under a severe weather

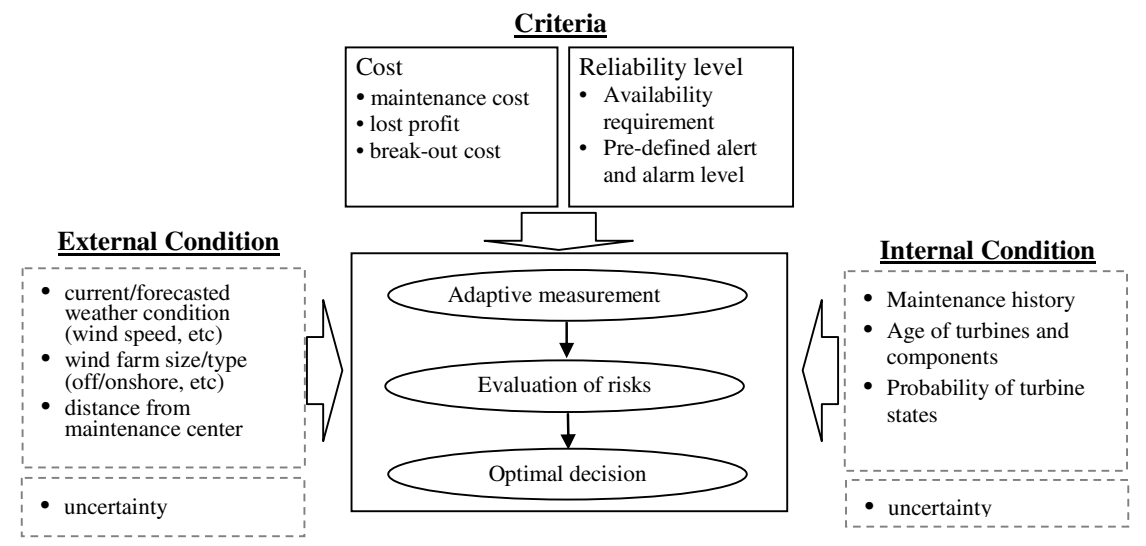

Fig. 4. Factors considered in the dynamic decision making process 
condition will be more costly than doing so under a fair condition. Shutting down the wind turbine for repair will also incur losses in power production so one would be better off to schedule such an action during a low wind period than a high wind period. In addition to the weather condition, a wind farm type such as where the wind farm is located (for instance, offshore will need special care and vessel), the distance between the wind farm and maintenance center are also the external factors in our decision making process.

As for the internal conditions related to the wind turbine, the probabilities about the turbine state, which is derived from sensor signals, are the most important input factor. Other factors include the age of the turbine and the repair history and so on. Our main objective in this decision making process is to minimize the risk caused by the information uncertainties $[10,11]$ from the external and internal factors. The criteria and influential factors are summarized in Fig. 4. Next, we present a simple example to explain the dynamic decision making process.

In the following example, we simplify the above consideration by assuming that the external conditions remain constant. We assume that a wind turbine could be in two health states: survival or failure and the sensor feedback can be classified into three categories: that is, $O_{t} \in\left\{o_{1}, o_{2}, o_{3}\right\}$ or $O_{t}^{\prime} \in\left\{o_{1}^{\prime}, o_{2}^{\prime}, o_{3}^{\prime}\right\}$, where $O_{t}^{\prime}$ is obtained from an advanced sensor under intensive care, and $o_{i}$ indicates an escalated risk of turbine failure as $i$ increases. Similar treatments were also used prior studies [8].

Fig. 5 shows a possible trajectory of sensory data and the corresponding scenario of decision rules at each epoch. The wind turbine operates normally during $t_{1} \sim t_{3}$. At $t_{4}, o_{2}$ is observed from a regular sensor, implying that an intensive care is needed. When invoking the advanced sensor, we got an $o_{1}{ }^{\prime}$ so we operate wind turbine as usual. Afterwards, $o_{2}$ and $o_{2}{ }^{\prime}$ are observed both at $t_{5}$ and $t_{6}$. Then we calculate the risk based on both sensor feedbacks and found the failure risk is not high enough at $t_{5}$ so we remain in the intensive care. But the failure risk crosses the alarm level at $t_{6}$ so we need to dispatch the crew for maintenance. After the repair, the wind turbine returns to the normal level. At $t_{10}$, an alarm signal is detected so we need to dispatch the crew right away without going through an intensive care period.

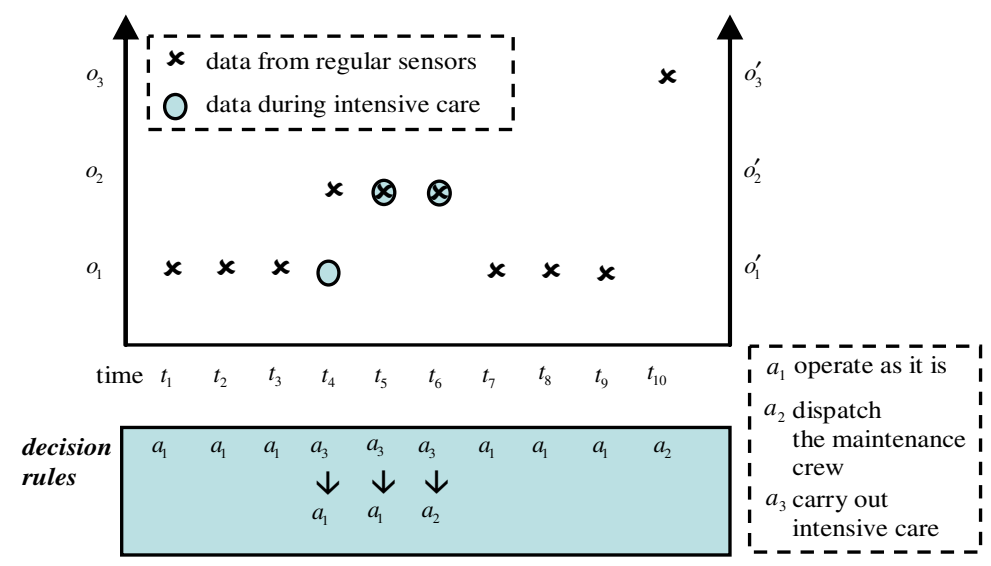

Fig. 5. A trajectory of sensor signals and decision rules 


\section{Summary}

This paper summarizes the recent progresses made in the dynamic data-driven fault diagnosis of wind turbine systems. The health monitoring of wind turbine systems is inherently challenging due to the high requirements placed on the accuracy and reliability of the monitoring system. To fundamentally solve the relevant issues, a series of tools and strategies have been developed and explored, which include robust data-driven local signal processing and preliminary detection algorithms, redundancy analysis for sensor network reliability and robustness, and global strategy for dynamic interrogation and decision making.

\section{Acknowledgements}

The authors gratefully acknowledge the support of the National Science Foundation through the grant number CMMI-0540132 and CMMI-0540278.

\section{References}

1. National Wind Technology Center, Wind Program Multi-Year Technical Plan, http://www.nrel.gov/wind_meetings/2003_imp_meeting/pdfs/mytp_nov_2003.pdf.

2. Zou, Y., Tong, L., and Steven, G.P.: Vibration-based model-dependent damage (delamination) identification and health monitoring for composite structures - a review. Journal of Sound and Vibration, 230 (2000) 357-378.

3. Tumer, I.R., and Huff, E.M.: Analysis of triaxial vibration data for health monitoring of helicopter gearboxes. ASME Journal of Vibration and Acoustics. 125 (2003) 120 - 128.

4. Newland, D.E.: Harmonic wavelet analysis. Proceedings: Mathematical and Physical Sciences. 443 (1993) 203-225.

5. Liu, B.: Adaptive harmonic wavelet transform with applications in vibration analysis. Journal of Sound and Vibration. 262 (2003) 45-64.

6. Konstantinides, K., and Yao, K.: Statistical analysis of effective singular value in matrix rank determination. IEEE Transactions on Acoustics, Speech \& Signal Processing. 36 (1988) 757-763.

7. Johnson, R.A., and Wichern, D.W.: Applied Multivariate Statistical Analysis, 5th Edition. Prentice Hall (2002).

8. Baruah P. and Chinnam, R. B.: HMMs for diagnosis and prognosis in machining process. International Journal of Production Research. 43 (2005) 1275-1293.

9. European Commission: Advanced maintenance and repair for offshore wind farms using fault prediction and condition monitoring techniques. Project report of European Commission under Contract NNE5/2001/710 (2005).

10. Gebraeel, N.: Sensory-updated residual life distributions for components with exponential degradation patterns. IEEE Transactions on Automation Science \& Engineering. 3 (2006) 382-393.

11. Pedregal, D.J. and Carnero, M. C.: State space models for condition monitoring: a case study. Reliability Engineering \& System Safety. 91 (2006) 171-180. 